\title{
Lithium chloride with immunomodulatory function for regulating titanium nanoparticle-stimulated inflammatory response and accelerating osteogenesis through suppression of MAPK signaling pathway
}

This article was published in the following Dove Press journal:

International Journal of Nanomedicine

\section{Chao Yang* \\ Wei Wang* \\ Kechao Zhu \\ Wei Liu \\ Yao Luo \\ Xiangwei Yuan \\ Jiaxing Wang \\ Tao Cheng (D) \\ Xianlong Zhang}

Department of Orthopedics, Shanghai Jiao Tong University Affiliated Sixth People's Hospital, Shanghai 200233,

People's Republic of China

*These authors contributed equally to this work
Correspondence: Xianlong Zhang; Tao Cheng

Department of Orthopedics, Shanghai Jiao Tong University Affiliated Sixth People's Hospital, Shanghai 200233,

People's Republic of China

$\mathrm{Tel}+862164369183$

Fax +86 216 470 I363

Email dr_zhangxianlong@163.com;

dr_tao.cheng@hotmail.com
Background: Wear particle-induced inflammatory osteolysis and the consequent aseptic loosening constitute the leading reasons for prosthesis failure and revision surgery. Several studies have demonstrated that the macrophage polarization state and immune response play critical roles in periprosthetic osteolysis and tissue repair, but the immunomodulatory role of lithium chloride $(\mathrm{LiCl})$, which has a protective effect on wear particle-induced osteolysis by suppressing osteoclasts and attenuating inflammatory responses, has never been investigated. Methods: In this work, the immunomodulatory capability of $\mathrm{LiCl}$ on titanium (Ti) nanoparticle-stimulated transformation of macrophage phenotypes and the subsequent effect on osteogenic differentiation were investigated. We first speculated that $\mathrm{LiCl}$ attenuated $\mathrm{Ti}$ nanoparticle-stimulated inflammation responses by driving macrophage polarization and generating an immune micro-environment to improve osteogenesis. Furthermore, a metal nanoparticle-stimulated murine air pouch inflammatory model was applied to confirm this protective effect in vivo.

Results: The results revealed that metal nanoparticles significantly activate M1 phenotype (proinflammatory macrophage) expression and increase proinflammatory cytokines secretions in vitro and in vivo, whereas $\mathrm{LiCl}$ drives macrophages to the $\mathrm{M} 2$ phenotype (antiinflammatory macrophage) and increases the release of anti-inflammatory and bone-related cytokines. This improved the osteogenic differentiation capability of rat bone marrow mesenchymal stem cells (rBMSCs). In addition, we also provided evidence that $\mathrm{LiCl}$ inhibits the phosphorylation of the p38 mitogen-activated protein kinase (p38) and extracellular signal-regulated kinase (ERK) pathways in wear particle-treated macrophages.

Conclusion: $\mathrm{LiCl}$ has the immunomodulatory effects to alleviate Ti nanoparticle-mediated inflammatory reactions and enhance the osteogenic differentiation of rBMSCs by driving macrophage polarization. Thus, $\mathrm{LiCl}$ may be an effective therapeutic alternative for preventing and treating wear debris-induced inflammatory osteolysis.

Keywords: lithium chloride, Ti nanoparticle, macrophage polarization, osteoimmunology, osteogenesis, immunomodulatory

\section{Introduction}

Total joint arthroplasty is one of the most successful surgical treatments for various endstage joint diseases. ${ }^{1}$ However, wear particle-induced aseptic loosening remains the leading reason for limiting the long-term survival of total joint arthroplasty. ${ }^{2,3}$ Wear particles, which come from the interface between the bone and the implant materials, 
play a crucial role in the particle-induced inflammatory cascade and consequent aseptic loosening. ${ }^{4-6}$ Previous studies have shown that wear debris are responsible for the stimulation of macrophages, which subsequently increase the production of proinflammatory factors, such as interleukin (IL)- 6 and tumor necrosis factor alpha (TNF- $\alpha)^{7,8}$ These proinflammatory cytokines can promote local inflammation by inducing the activation and differentiation of macrophages to a proinflammatory phenotype (M1 macrophages). Recently, the concept of macrophage polarization was supposed to play a crucial role in the process of wear particle-induced inflammatory osteolysis. ${ }^{9}$ M1 macrophage regulation of proinflammatory cytokine (IL-6, TNF- $\alpha$ ) secretion is well known, and recognized to enhance osteoclastic activities and exert immunostimulatory effects. ${ }^{10-12}$ By contrast, anti-inflammatory phenotypes, M2 macrophages, enhance tissue repair by secreting relevant cytokines (IL-4, IL-10, vascular endothelial growth factor [VEGF], and bone morphogenetic protein [BMP]-2) which contribute to the process of osteogenesis. ${ }^{13,14}$ Some studies have already indicated that the local microenvironmental conditions induced by M2 macrophages is beneficial for osseointegration and angiogenesis; this is known as osteoimmunology. ${ }^{15-17}$ Thus, the regulation of macrophage polarization is deemed an effective strategy to alleviate wear particle-induced inflammatory osteolysis and enhance osseointegration of implants.

Lithium chloride $(\mathrm{LiCl})$ is one of the currently prescribed drugs for treating patients with bipolar disorder and epilepsy. Even though many studies have indicated that $\mathrm{LiCl}$ can regulate some biological processes, such as inflammation, apoptosis, and glycogen synthesis, the mechanisms involved in these processes are not well understood. ${ }^{18-20}$ Furthermore, it has been reported that $\mathrm{LiCl}$ enhances osteogenesis in wear particle-induced osteolysis via Glycogen synthase kinase-3 $\beta$ (GSK-3 $\beta$ ) signaling pathway inhibition and attenuates osteoclastogenesis by suppressing the nuclear factor-kappa B (NF$\kappa \mathrm{B})$ pathway. ${ }^{19,21}$ In addition to these biological processes, the effects of $\mathrm{LiCl}$ in regulating inflammation in different immune cell models, especially macrophages, in the context of inflammatory diseases, have also been explored. ${ }^{22-24}$ However, until now, not much was known about the immunomodulatory effect of $\mathrm{LiCl}$ on macrophage polarization and its subsequent influence on the osteogenic differentiation potential of mesenchymal stem cells.

Therefore, the purpose of this study was to investigate the potential immunomodulatory capacity of $\mathrm{LiCl}$ on wear particle-treated macrophages and its subsequent impact on osteogenic differentiation in vitro and in vivo. Interestingly,
$\mathrm{LiCl}$ alleviated titanium (Ti) particle-induced inflammation and promoted osteogenesis in a conditioned medium. Furthermore, the results of this study suggest that these immunomodulatory effects may be attributed to the regulation of macrophage polarization in the local microenvironment through the inhibition of the p38 mitogen-activated protein kinase (p38) and extracellular signal-regulated kinase (ERK) phosphorylation.

\section{Materials and methods}

\section{Ti particles}

Ti particles (99.99\% purity) were obtained from Johnson Matthey Chemical (MA, USA). Ti particles were endotoxinnegative, as verified by a limulus amebocyte lysate assay. ${ }^{25}$ The Ti particles characteristics were observed by a transmission electron microscope (TEM). The particle diameters were determined by ImageJ software as previously described. ${ }^{26}$

\section{Cell culture}

RAW264.7 macrophages were obtained from the Type Culture Collection of the Chinese Academy of Sciences (Shanghai, China), whereas rat bone marrow mesenchymal stem cells (rBMSCs) were isolated and cultured as previously described. ${ }^{27}$ RAW264.7 macrophages and rBMSCs were cultured in Dulbecco's Modified Eagle's Medium (DMEM, HyClone), containing 10\% fetal bovine serum (Gibco) and $1 \%$ penicillin/streptomycin (HyClone) at $37^{\circ} \mathrm{C}$ in $5 \% \mathrm{CO}_{2}$. The cells were seeded onto plates before stimulation with or without Ti particles $(0.1 \mathrm{mg} / \mathrm{mL})$ or treatment with a combination of wear particles and different concentrations of $\mathrm{LiCl}(0,1$ or $5 \mathrm{mM})$. The medium was replaced every 2 days. Images of RAW264.7 macrophages were photographed via a light microscope (Leica).

\section{Cell proliferation assay}

Cell proliferation was analyzed using a Cell Counting Kit8 (CCK-8) Assay. RAW264.7 cells were seeded onto a plate at $5 \times 10^{4}$ per well, whereas $\mathrm{rBMSCs}$ were seeded at $2.5 \times 10^{4}$ per well. The cells were washed thrice and $10 \%$ CCK-8 containing medium was added for $4 \mathrm{~h}$ at $37^{\circ} \mathrm{C}$. Then a microplate reader was used to measure the absorbance at $450 \mathrm{~nm}$.

\section{Immunofluorescent staining of macrophage polarization}

Immunofluorescence staining was performed to assess the activation of the C-C chemokine receptor type 7 (CCR7, 
M1 phenotype) and arginase-1 (Arg-1, M2 phenotype). After culturing for 4 days, the cells were fixed in paraformaldehyde $(4 \%)$, then blocked with $1 \%$ bovine serum albumin (BSA) for $30 \mathrm{~min}$ and cultured with CCR7 (1:100, Abcam) and Arg-1 (1:100, Abcam) antibodies overnight at $4^{\circ} \mathrm{C}$. The next day, the cells were rinsed with PBS, treated with the secondary antibodies, donkey anti-mouse Alexa Fluor 594 (1:200, Abcam) and donkey anti-rabbit Alexa Fluor 488 (1:200, Abcam), and incubated for $1 \mathrm{~h}$ in the dark. Then, 4',6-diamidino-2-phenylindole (DAPI) was used to stain cell nuclei for $10 \mathrm{~min}$.

\section{Flow cytometry}

The expression of the M1 marker, CCR7, and M2 marker, cluster of differentiation 206 (CD206) were analyzed by flow cytometry. After culturing for 4 days, cells were scraped, washed, and resuspended in $1 \%$ BSA for $30 \mathrm{~min}$. Then, they were treated with allophycocyaninconjugated CCR7 (eBioscience) and phycoerythrin-conjugated CD206 (eBioscience) antibodies for $1 \mathrm{~h}$. Moreover, allophycocyanin-conjugated Armenian hamster immunoglobulin $\mathrm{G}$ and phycoerythrin-conjugated rat immunoglobulin G2a, $\kappa$, were used as isotype control. Guava flow cytometer and software (Millipore, USA) were used to analyze the results.

\section{Enzyme-linked immunosorbent assay (ELISA)}

After culturing for 4 days, the cell medium was collected and centrifuged. The concentrations of TNF- $\alpha$, IL-4, IL-6, and IL-10 in the supernatants were determined using ELISA kits (Anogen, Canada) according to the manufacturer's instructions.

\section{Real-time polymerase chain reaction (RT- PCR)}

Gene expression of the M1 macrophage marker CD86, the M2 macrophage marker CD163, and the bone-related gene BMP-2 and VEGF were quantified via RT-PCR. After 4 days of culture, TRIzol reagent (Invitrogen) was used to extract total RNA. Complementary DNA was synthesized from $1 \mu \mathrm{g}$ of total RNA using a RevertAid First Strand cDNA Synthesis kit (Thermo). Quantitative gene analysis was performed using FastStart Universal SYBR Green Master (Rox, Roche) and a thermocycler instrument (ABI). The primers used in this section are shown in Table 1.
Table I Primers for RT-PCR used to quantify the expression of RAW264.7

\begin{tabular}{|l|l|l|}
\hline Gene & $\begin{array}{l}\text { Primer sequences (F: forward; } \text { R: } \\
\text { reverse; 5'-3') }\end{array}$ & $\begin{array}{l}\text { Length } \\
\text { (bp) }\end{array}$ \\
\hline CD86 & $\begin{array}{l}\text { F: TGGGCGCAGAGAAACTTGAT } \\
\text { R: AAGCCCGTGTCCTTGATCTG }\end{array}$ & 127 \\
\hline CDI63 & $\begin{array}{l}\text { F: GTGGTCAACTCCGCTTGGTA } \\
\text { R: CTTGGGGCACCATCTGTGAT }\end{array}$ & 126 \\
\hline BMP-2 & $\begin{array}{l}\text { F: AACGAGAAAAGCGTCAAGCC } \\
\text { R: AGGTGCCACGATCCAGTCAT }\end{array}$ & 135 \\
\hline VEGF & $\begin{array}{l}\text { F: GCAAGAGAAGACACGGTGGT } \\
\text { R: CAGGAGGTGGGGTAAGGAG }\end{array}$ & $\begin{array}{l}\text { F: AAATGGTGAAGGTCGGTGTG } \\
\text { R: AGGTCAATGAAGGGGTCGTT }\end{array}$ \\
\hline
\end{tabular}

\section{Western blot analysis}

The cells were pretreated with $5 \mathrm{mM} \mathrm{LiCl}$ for $4 \mathrm{~h}$. Ti particles were then added to the medium at specific times. Afterwards, the cells were lysed for protein extraction in radioimmunoprecipitation assay (RIPA) lysis buffer, containing protease and phosphatase inhibitors (Yeasen, Shanghai), for $30 \mathrm{~min}$ on ice. Total cell protein was separated by sodium dodecyl sulfate-polyacrylamide gel electrophoresis and subsequently transferred to polyvinylidene fluoride membranes. The membranes were blocked in 5\% skim milk for $1 \mathrm{~h}$, and probed with the relevant antibodies: ERK, p-ERK, p38, p-p38, and $\beta$ actin. After washing thrice with Tris-buffered saline and Tween, the membranes were incubated with secondary antibodies for $1 \mathrm{~h}$. Finally, the protein bands were developed with an enhanced chemiluminescence agent (Millipore, China). The relative gray levels of p-ERK and p-p38 were quantified and normalized to $\beta$-actin using ImageJ software.

\section{Osteogenic differentiation effect of rBMSCs in conditioned medium}

\section{Preparation of conditioned medium}

After 4 days of culture, conditioned medium was collected and the supernatants from the culture media were mixed with complete medium at a $1: 2$ ratio. ${ }^{10}$ Next, rBMSCs were seeded onto a plate at a density of $2 \times 10^{4}$ cells per well. After incubating for $12 \mathrm{~h}$, the conditioned medium was used for further incubation. 
Alkaline phosphatase (ALP) and alizarin red staining (ARS)

After culturing for 2 weeks, the cells were fixed and subjected to ALP or ARS dye (Beyotime). ALP activity was measured using the ALP kit (Beyotime) after 14 days and the values were normalized against the protein concentration, which was determined using a bicinchoninic acid protein assay kit (Beyotime). In addition, quantitative analysis of ARS was initiated by the addition of $10 \%$ cetylpyridinium chloride and the optical density was measured using a microplate reader at $600 \mathrm{~nm}$.

\section{Immunofluorescent staining}

Immunofluorescent staining of ALP was also used to assess osteogenic differentiation. After culturing in conditioned medium for a specific period, rBMSCs were fixed and permeabilized with $0.1 \%$ Triton-X for $15 \mathrm{~min}$, then blocked with $1 \%$ BSA and incubated with ALP (1:100, Abcam) antibodies overnight at $4{ }^{\circ} \mathrm{C}$. The next day, the cells were rinsed with PBS and incubated with the secondary antibody for $1 \mathrm{~h}$. Then, phalloidin and DAPI were used to stain the cytoskeleton and nuclei, respectively, for $10 \mathrm{~min}$.

\section{In vivo mouse air pouch model}

The animal experiment was approved by the Animal Care and Experiment Committee of Sixth People's Hospital affiliated with Shanghai Jiao Tong University. Animal care and use were conducted according to the policies of the Institutional Animal Care and Use Committee of Shanghai Jiao Tong University, the regulations for the Administration of Affairs Concerning Experimental Animals (China, 2014), and the National Institutes of Health Guide for the Care and Use of Laboratory Animals (GB14925-2010). C57BL/6 mice were used for the experiment as previously described. ${ }^{28}$ After air pouch formation, pouches were injected with $0.5 \mathrm{~mL}$ PBS alone or $0.5 \mathrm{~mL}$ PBS containing Ti particles with or without different concentrations of $\mathrm{LiCl}$. After 4 days of injection, the mice were sacrificed and the air pouches were washed with $2 \mathrm{~mL}$ PBS. The exudates were collected, centrifuged and stored at $-80^{\circ} \mathrm{C}$ for ELISA as previously described. ${ }^{9}$ Finally, the air pouch was harvested and collected for further histological analysis. In addition, the heart, kidney, liver, and lung of mice were also harvested and fixed to evaluate the bio-safety of $\mathrm{LiCl}$ on major organs. Sections of major organs were embedded, cut and subjected to hematoxylin and eosin staining.
The air pouch membranes were embedded and cut into $4-\mu \mathrm{m}$ sections using a microtome. Then, the tissue was subjected to hematoxylin and eosin and Masson trichrome staining to assess the inflammatory reaction of the pouch membranes. The thickness and cell infiltration of the pouch membranes were measured via the Image-Pro Plus software, and other tissue sections were subjected to immunofluorescence staining to assess the expression of CCR7 and Arg-1-positive cells as described above. ${ }^{14,29}$

\section{Statistical analysis}

All the data were analyzed by SPSS 17.0 software and expressed as the mean \pm standard deviation (SD). Differences among groups were analyzed with one-way ANOVA followed by the Student's $t$-test were used to evaluate the significance of differences. Differences with $P<0.05$ were considered statistically significant.

\section{Results}

\section{Particles characterization}

The morphology of the Ti particles was observed by TEM and the characteristics of Ti particles are shown in Figure 1A and B. Most Ti particles had irregular morphology, and almost $80 \%$ of the particles ranged from 30 to $90 \mathrm{~nm}$ with a mean diameter of $52.59 \pm 20.48 \mathrm{~nm}$ (Figure 1C). These characteristics were similar to those of previously described wear particles. ${ }^{30,31}$

\section{Cell morphology and proliferation}

Light microscopy and CCK-8 assay were used to assess cell morphology and proliferation, and the results are depicted in Figure 2. In the control group, RAW cells, unstimulated by wear particles, were round and small. However, the cells stimulated by $\mathrm{Ti}$ particles were flat with many synaptic structures. Polygonal cells decreased in groups treated with $\mathrm{LiCl}$, and cone-shaped cells increased in the group treated with high $\mathrm{LiCl}$ concentration (Figure 2A). The results of cell proliferation are presented in Figure 2B and C. After incubation for 1 and 4 days, $\mathrm{LiCl}$ and $\mathrm{Ti}$ particles were not obviously cytotoxic to RAW264.7 and rBMSCs.

\section{In vitro macrophage polarization}

After culturing for 4 days, the expression of CCR7 (green) and Arg-1 (red) in RAW264.7 cells were assessed by immunofluorescence staining. As shown in Figure 3, the trend of Arg-1 expression was: $\mathrm{Ti}<\mathrm{Control}<\mathrm{Ti}+\mathrm{LiCl1}<\mathrm{Ti}+\mathrm{LiCl} 5$. The result of immunofluorescence staining indicated that the 
A

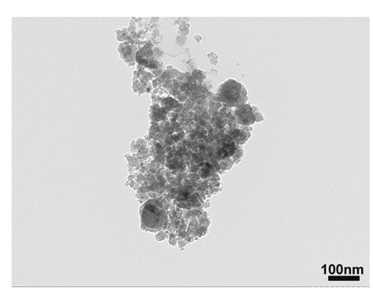

B

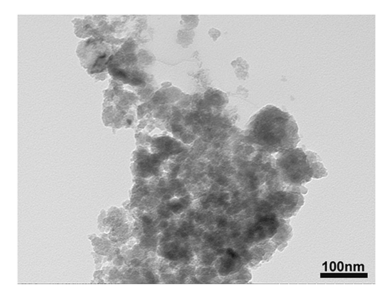

C

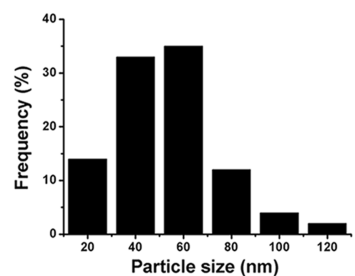

Figure I Characterization of Ti particles.

Notes: $(\mathbf{A})$ and $(\mathbf{B})$ Representative TEM image of Ti particles. (C) Ti particles size distribution, particles with sizes of $52.59 \pm 20.48 \mathrm{~nm}$ (mean \pm SD). Scale bar $=100 \mu \mathrm{m}$. Abbreviations: Ti, titanium; TEM, transmission electron microscopy; SD, standard deviation.

\section{A}

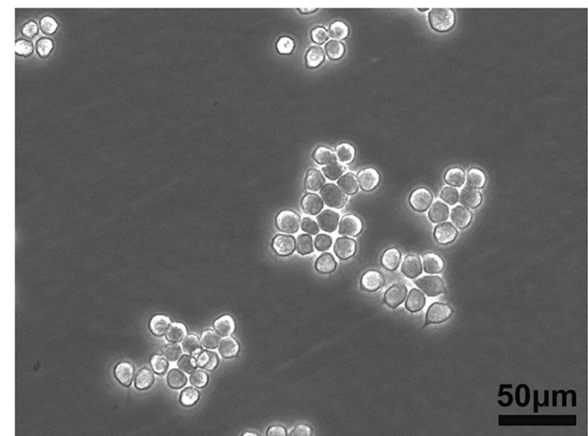

Contorl

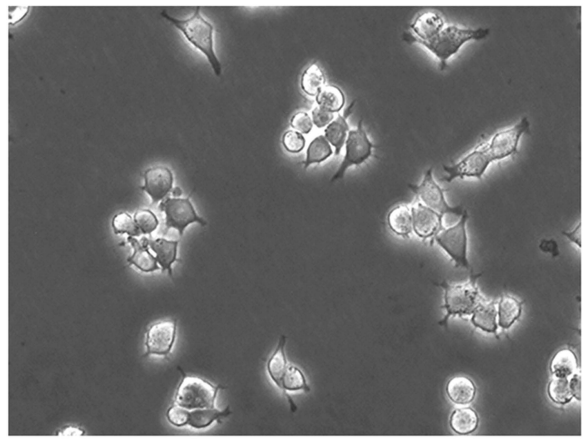

$\mathrm{Ti}+\mathrm{LiCl1}$

B

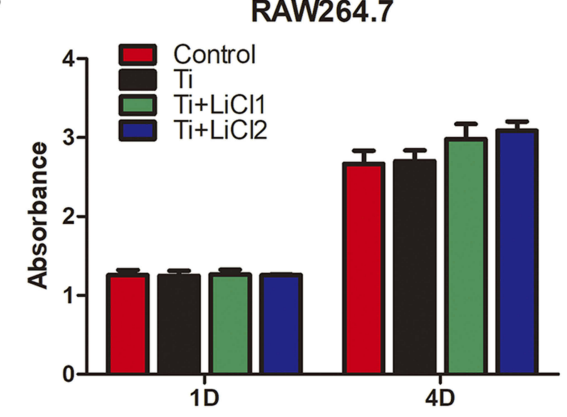

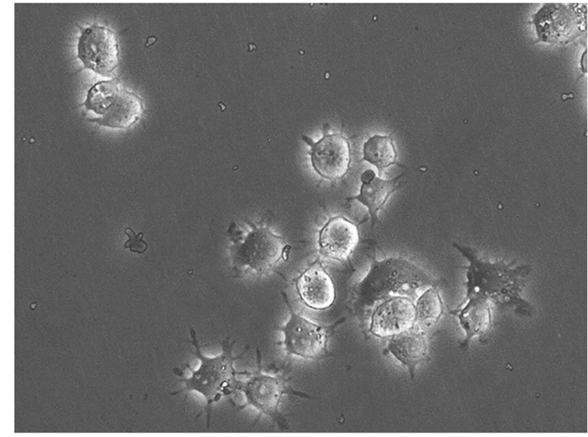

$\mathrm{Ti}$

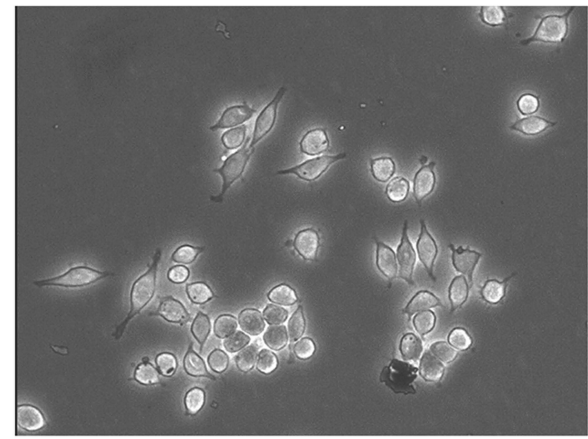

$\mathrm{Ti}+\mathrm{LiCl5}$

C

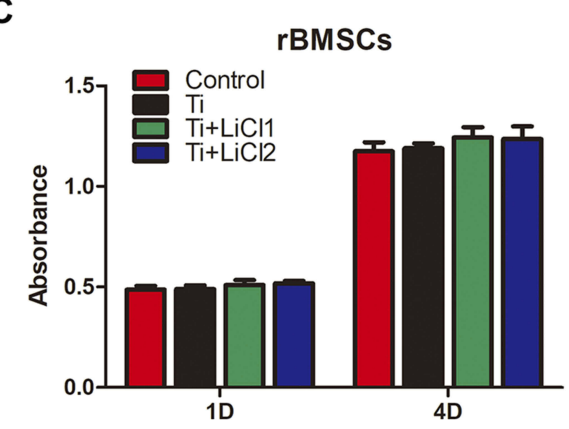

Figure 2 Cell morphology and proliferation.

Notes: (A) Morphology of RAW macrophages obtained by a light microscope. Scale bar: $50 \mu \mathrm{m}$. (B) and (C) Cell proliferation was evaluated by CCK-8 after I and 4 days of culture.

Abbreviation: CCK-8, cell counting kit-8.

groups treated with $\mathrm{LiCl}$ featured a higher expression of the however, indicated a contrary trend: more CCR7-positive M2 phenotype than the control and Ti particle groups. CCR7, cells were detected in the Ti particle group. 


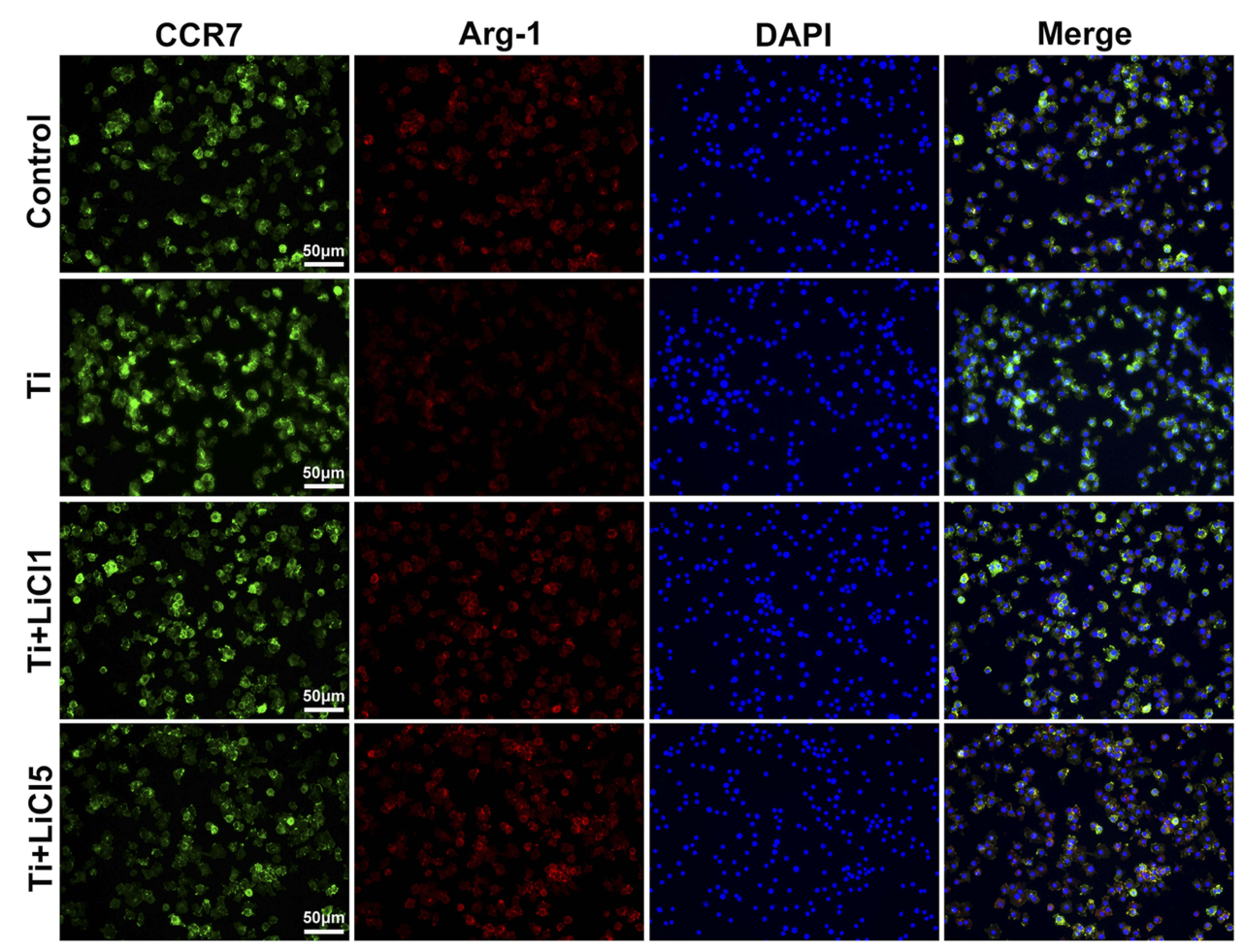

Figure 3 Immunofluorescence staining of RAW cells.

Notes: CCR7 (green fluorophore) indicates MI macrophages; Arg-I (red fluorophore) indicates M2 macrophages; nuclei are stained with DAPI (blue fluorophore), scale bar: $50 \mu \mathrm{m}$.

Abbreviations: CCR7, C-C chemokine receptor type 7; Arg-I, arginase-I; DAPI, 4',6-diamidino-2-phenylindole.

Flow cytometry was used to quantify the percentage of CCR7 and CD206-positive cells simultaneously. Representative histograms of CCR7 and CD206 are shown in Figure $4 \mathrm{~A}$ and $\mathrm{B}$ and the dot plot of RAW cells is depicted in Figure 4C. The percentage of CCR7 and CD206 are presented in Figure 4D and E. The results shown that the expression of M1-positive cells reduced from $79.27 \%$ in the $\mathrm{Ti}$ group to $41.99 \%$ in the $\mathrm{Ti}+\mathrm{LiCl} 5$ group. In addition, the expression of M2-positive cells was higher in the $\mathrm{Ti}+\mathrm{LiCl} 5$ group $(47.19 \%)$ than in the $\mathrm{Ti}$ $(6.87 \%)$ and control (14.19\%, Figure 4B and E) groups.

ELISA was performed to detect cytokine secretion levels and RT-PCR was performed to determine the expression of related genes. As depicted in Figure 5A-D, macrophages in the $\mathrm{Ti}+\mathrm{LiCl} 5$ group released the highest amounts of IL-4 and IL-10, which are mainly generated by anti-inflammatory macrophages. However, the secretion of inflammatory cytokines, TNF- $\alpha$ and IL-6, were the highest in the Ti group. The M1 phenotype marker gene, CD86, was downregulated in the $\mathrm{LiCl}$-treated groups compared to the control and Ti particle groups (Figure 5E). However, the expression of marker gene, CD163, representing M2 macrophages, was elevated in the LiCl-treated groups and not in the other groups (Figure 5F). In addition, the expression levels of BMP-2 and VEGF (bone-related gene) were also upregulated in the $\mathrm{Ti}+\mathrm{LiCl} 5$ group (Figure $5 \mathrm{G}$ and $\mathrm{H}$ ), suggesting a potential osteogenic capacity in the LiCltreated groups.

\section{$\mathrm{LiCl}$ attenuates Ti particle-induced activation of ERK and p38 phosphorylation in vitro}

Previous studies have reported that poly (methyl methacrylate) and hydroxyapatite particles stimulate the activation of the ERK and p38 pathways, and drive M1 macrophage polarization. ${ }^{32}$ However, whether Ti particles and $\mathrm{LiCl}$ are involved in the mitogen-activated protein kinase (MAPK) pathway, or not, is unknown. In this study, RAW cells were stimulated with different concentration of Ti particles over the course of $30 \mathrm{~min}$, then, ERK and $\mathrm{p} 38$ activation, as indicated by phosphorylation, was measured by Western blot analysis (Figure 6), which revealed that ERK and p38 phosphorylation were significantly increased by Ti particles stimulation (Figure 6A-C). As presented in Figure 6D-F, the activation of ERK and p38 was reduced by $\mathrm{LiCl}$ treatment compared to $\mathrm{Ti}$ treatment, indicating that $\mathrm{LiCl}$ attenuated wear particle-induced 
A

B
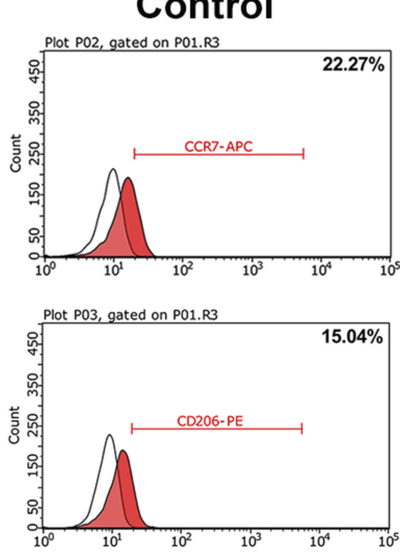

C

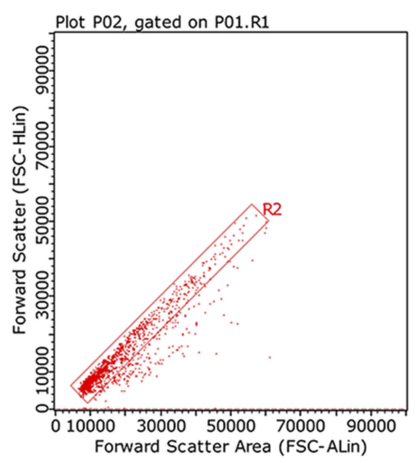

Ti
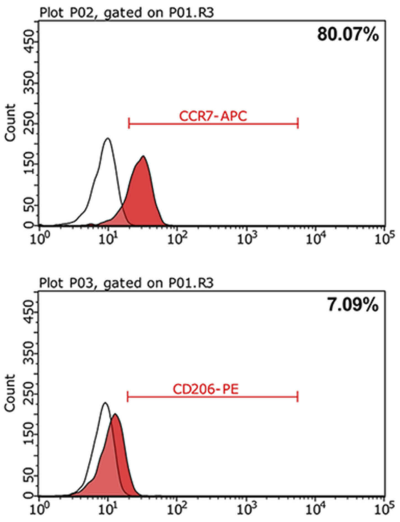

D
Ti+LiCl1
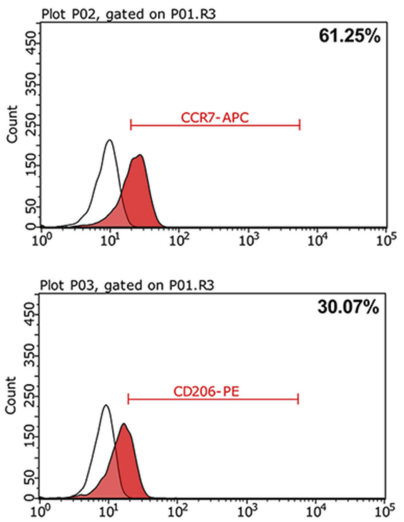

Ti+LiCl5
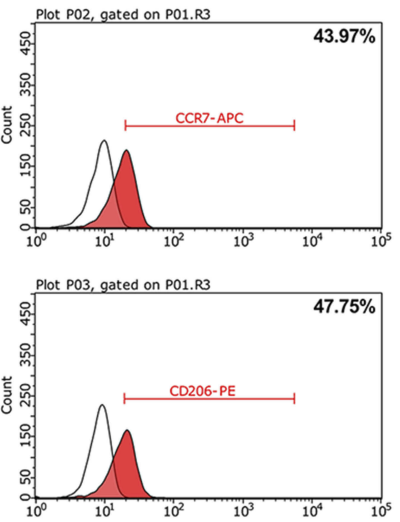

E
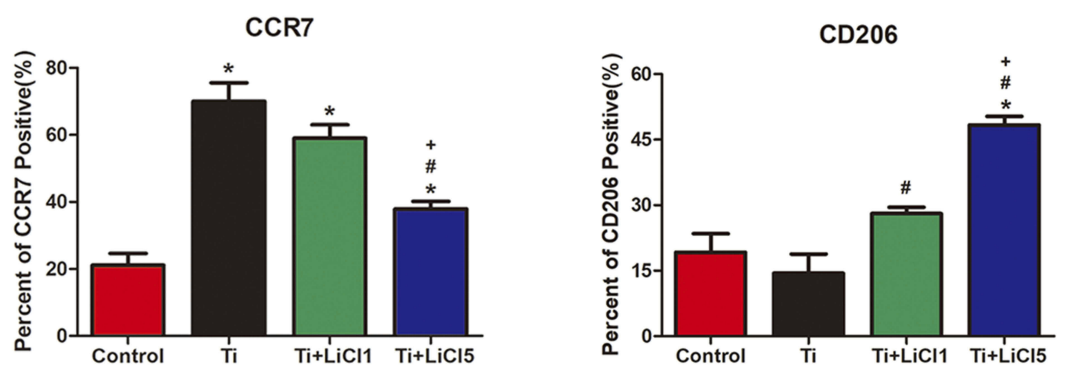

Figure 4 In vitro polarization of macrophage.

Notes: (A) and (B) Representative histograms of flow cytometry results after cultured for 4 days, percentage of CCR7 and CD206 positive cells, representing MI or M2 macrophages, respectively. (C) Flow cytometry analysis of RAW264.7 cells. (D) and (E) Percentage of CCR7 and CD206 positive cells respectively. (*\# and + represent $\mathrm{P}<0.05$ when compared with Control, $\mathrm{Ti}$, and $\mathrm{Ti}+\mathrm{LiClI}$ respectively).

Abbreviations: CCR7, C-C chemokine receptor type 7; CD206, cluster of differentiation 206.

A

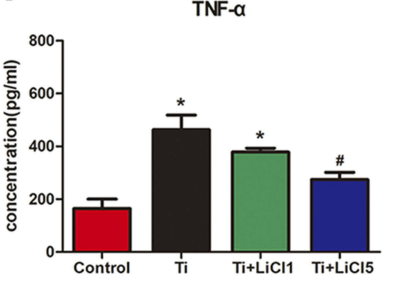

B

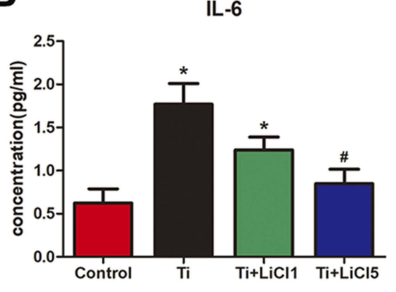

C

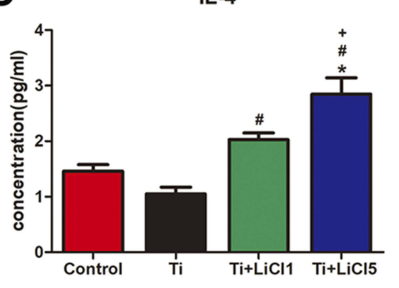

D

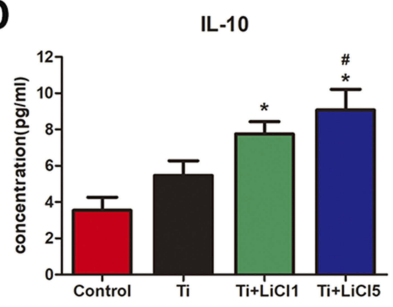

E

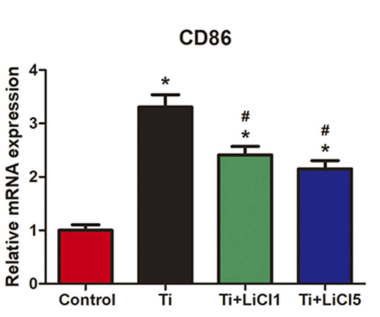

$\mathbf{F}$

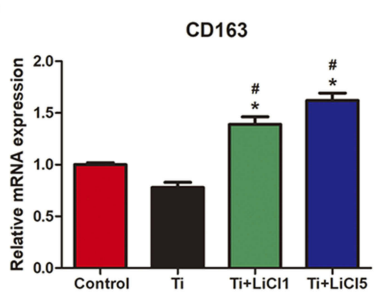

G

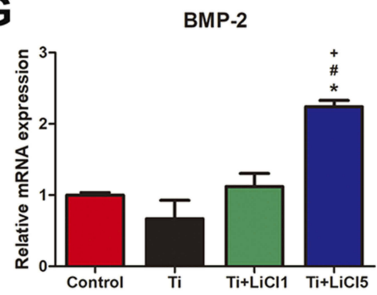

H

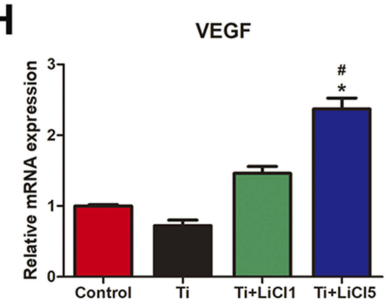

Figure 5 ELISA determination of cytokine, RT-PCR analysis of genes from RAW cells.

Notes: (A) TNF- $\alpha,(\mathbf{B})$ IL-6, (C) IL-4, (D) IL-10, (E) Gene expression of MI marker CD86, (F) Gene expression of M2 marker CDI63, (G) BMP-2 and (H) VEGF. (*\# and + represent $P<0.05$ when compared with Control, Ti, and $\mathrm{Ti}+\mathrm{LiClI}$ respectively).

Abbreviations: ELISA, enzyme-linked immunosorbent assay; RT-PCR, real-time polymerase chain reaction; TNF- $\alpha$, tumor necrosis factor alpha; IL, interleukin; CD, cluster of differentiation; BMP-2, bone morphogenetic protein-2; VEGF, vascular endothelial growth factor. 
A

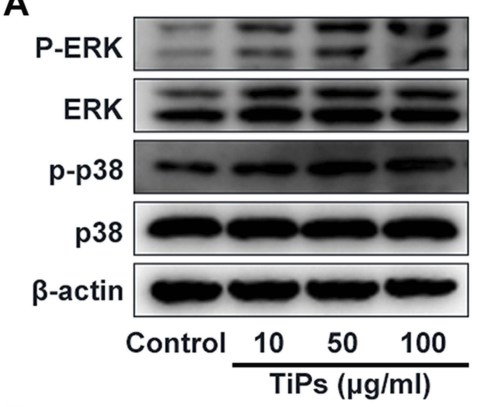

D

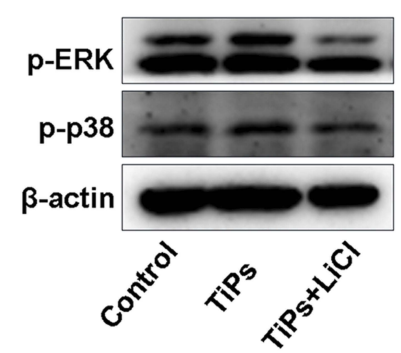

B

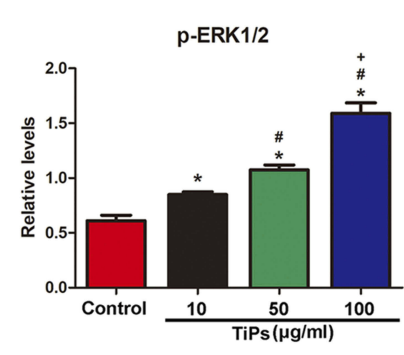

E

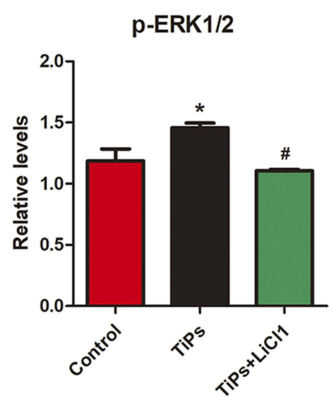

C

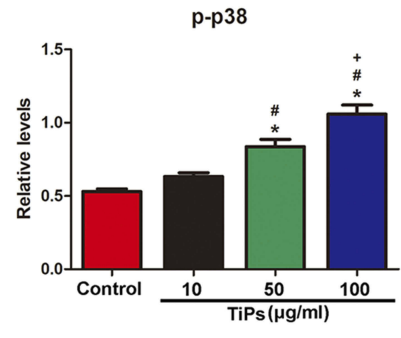

F

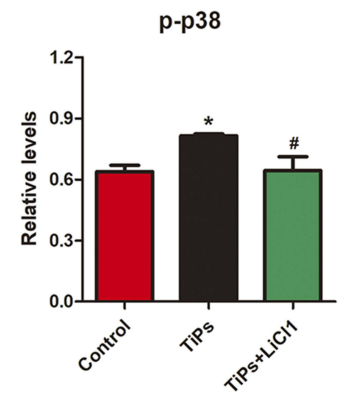

Figure $6 \mathrm{LiCl}$ attenuates Ti particle-induced the activation of ERK and p38 phosphorylation in RAW cells.

Notes: (A) Western blots performed after macrophages were treated with various concentrations $(0,10,50,100 \mu \mathrm{g} / \mathrm{mL})$ of Ti particles for the indicated times. (B) and $(\mathbf{C})$ The density of Western blot bands shown in (A) was quantified using Image software. (D) Western blots performed after macrophages were treated with PBS (control), Ti particles or Ti particles $+\mathrm{LiCl}$. (E) and (F) The density of Western blot bands shown in (D) was quantified using Imagej software. $(* \#$ and + represent $P<0.05$ when compared with Control, $\mathrm{Ti}$, and $\mathrm{Ti}+\mathrm{LiClI}$ respectively).

Abbreviations: $\mathrm{LiCl}$, lithium chloride; $\mathrm{Ti}$, titanium; ERK, extracellular signal-regulated kinase.

inflammation via the suppression of ERK and p38 phosphorylation in vitro.

\section{The evaluation of osteogenic differentiation capacity in conditioned medium}

RAW cell culture supernatant was used as conditioned medium to evaluate its effect on osteogenic differentiation of rBMSCs. The results of ALP and ARS staining demonstrated a higher level of ALP expression and extracellular matrix mineralization $(\mathrm{ECM})$ in the $\mathrm{Ti}+\mathrm{LiCl} 5$ group, followed by the $\mathrm{Ti}+\mathrm{LiCl1}$ group (Figure 7A and B). The ALP activity and optical density values of ECM showed similar results (Figure 7C and D). Consistent with ALP and ARS staining, a similar trend was detected in immunofluorescence staining: the $\mathrm{Ti}+\mathrm{LiCl} 5$ group had a higher ALP fluorescence intensity than other groups, and the lowest ALP fluorescence intensity was observed in the Ti group (Figure 7E).

\section{In vivo air pouch model}

To assess the inflammatory response and different macrophages phenotypes infiltrate the skin of the air pouch, we carried out histological and immunofluorescent staining of the skin sections. The hematoxylin and eosin- and Masson trichrome- staining sections are shown in Figure 8A and D. The blue arrows represent the fibrous layer. The results of histological staining suggested that air pouches treated with wear particles provoked a pronounced inflammatory reaction, presenting as increased thickness and cell infiltration of the pouch membrane compared to other groups. In contrast to Ti groups, $\mathrm{LiCl}$ attenuated the inflammatory reaction by decreasing the thickness of the fibrous layer and cell infiltration. The general observation was consistent with the quantitative results (Figure 8B and C). As shown in Figure $8 \mathrm{E}-\mathrm{H}$, the concentration of proinflammatory cytokines (TNF- $\alpha$ and IL-6) in the LiCl-treated groups were lower than those in the Ti group. However, the anti-inflammatory cytokines, IL-4 and IL-10, were upregulated in the exudates injected with $\mathrm{LiCl}$. The ELISA results from in vivo experiments, were in accordance with those in vitro. Additionally, an in vivo bio-safety assay of major organs confirmed that $\mathrm{LiCl}$ attenuated the inflammatory responses by regulating the ratio of M1/M2 macrophages that were non-toxic to key organs (Figure S1).

The expression of CCR7 (green, yellow arrows) and Arg-1 (red, white arrows) in RAW264.7 cells were also 

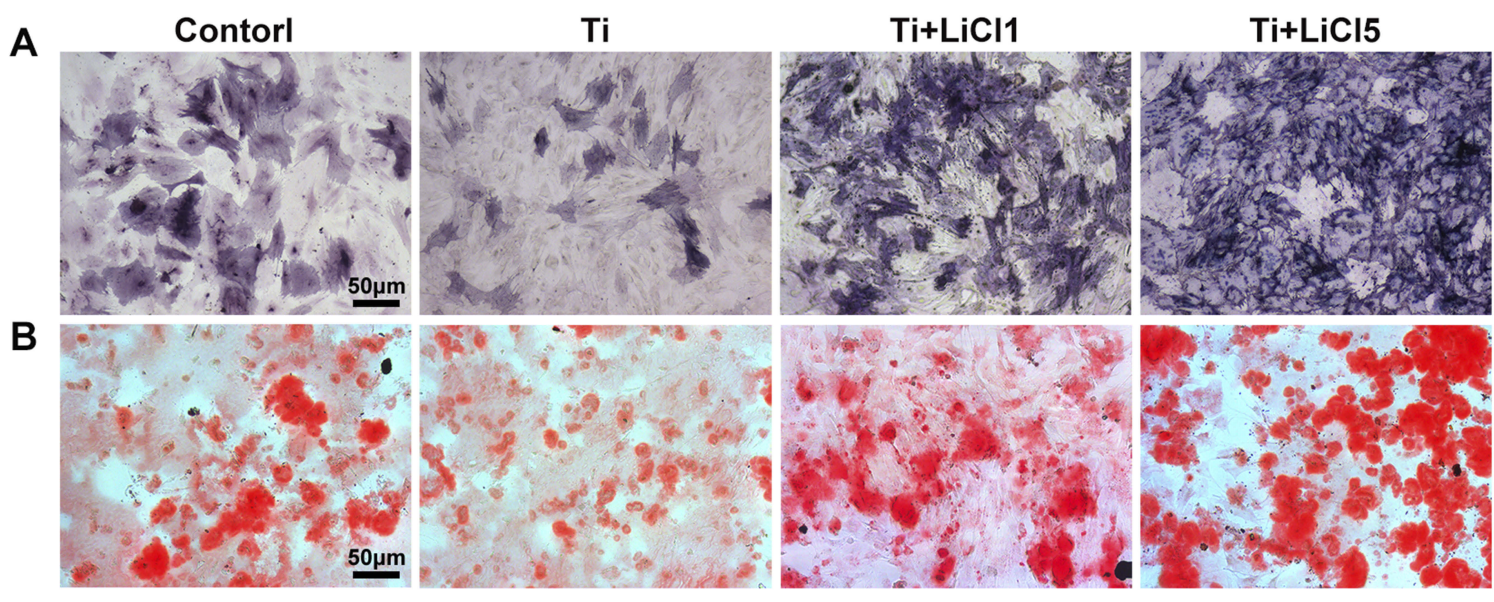

C

E

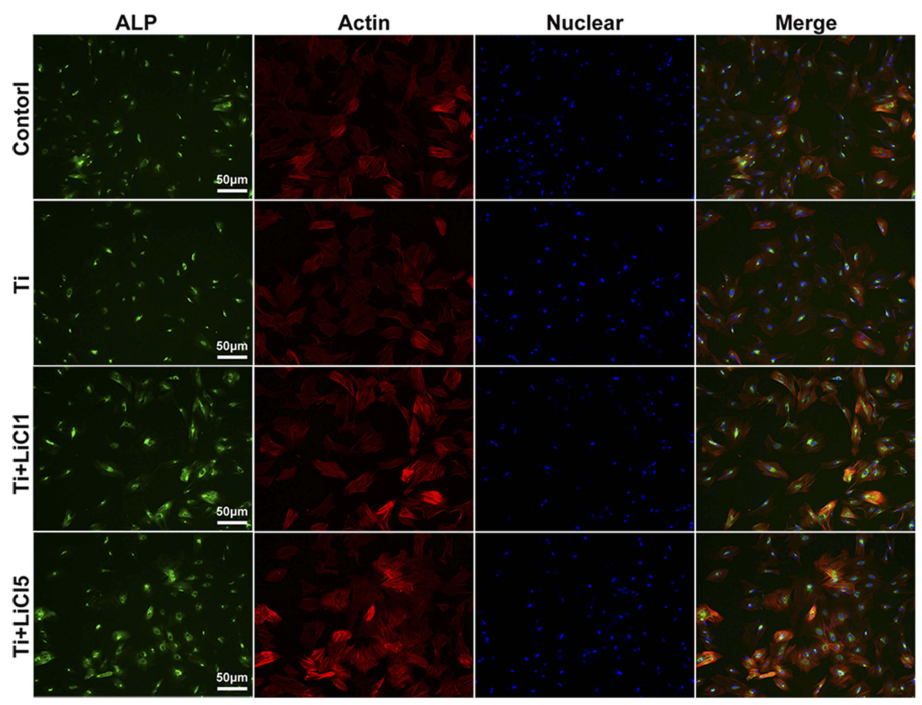

Figure 7 Osteogenic differentiation effect of macrophage-conditioned medium.

Notes: (A) ALP staining and (B) ARS staining of rBMSCs cultured in conditioned medium for 14 days. (C) ALP activity of rBMSCs cultured in conditioned medium for I4 days. (D) Quantitative analysis of Alizarin red staining. (E) ALP immunofluorescent staining of rBMSCs cultured in conditioned medium: ALP (green), actin (red), nuclear (blue). scale bar: $50 \mu \mathrm{m}$. (*\# and + represent $\mathrm{P}<0.05$ when compared with Control, $\mathrm{Ti}$, and Ti+LiCll respectively).

Abbreviations: ALP, Alkaline phosphatase; ARS, alizarin red staining; rBMSCs, rat bone marrow mesenchymal stem cells.

assessed by immunofluorescence staining in vivo. The blue arrows show the fibrous layer and skin (Figure 9). As shown in Figure 9, more CCR7 positive cells were observed in the Ti particle group, but not in other groups; in contrast, the $\mathrm{Ti}+\mathrm{LiCl} 5$ group featured a higher proportion of Arg-1 positive cells. Therefore, the in vivo experimental results were highly consistent with the results from the in vitro experiments, indicating that $\mathrm{LiCl}$ can drive macrophage polarization and induce an osteogenic immune microenvironment.

\section{Discussion}

Total joint arthroplasty is one of the most successful treatment strategies to be developed in past decades; however, wear debris, generated from orthopedic materials, can stimulate host immune cells to enhance inflammatory responses and induce osteolysis. ${ }^{33,34}$ The wear particles are preponderantly phagocytosed by macrophages, which produce various inflammatory chemokines and cytokines. ${ }^{31}$ Specifically, several studies have reported that the state of macrophage polarization may affect inflammatory response and generate an osteogenic immune microenvironment. ${ }^{10,35,36}$ Recently, $\mathrm{LiCl}$ has been studied in relation to various bone lossrelated diseases and inhibited inflammatory cytokine expression, especially in wear debris-induced inflammatory response in vivo. ${ }^{21,37}$ Moreover, the immune-modulatory properties of $\mathrm{LiCl}$ have also drawn much 
A

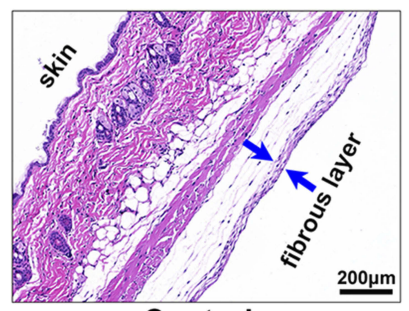

Contorl

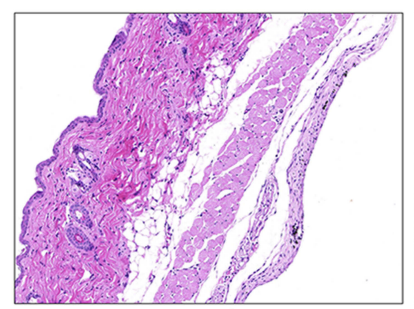

$\mathrm{Ti}+\mathrm{LiCl} 1$

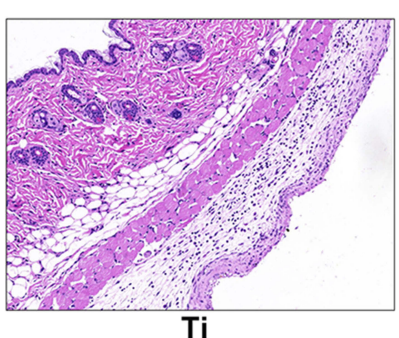

$\mathrm{Ti}$

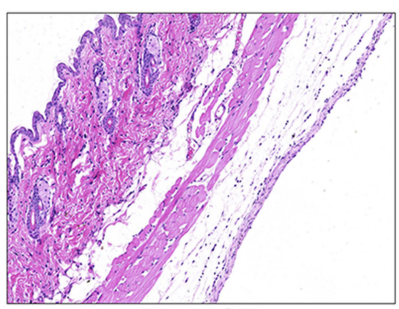

$\mathrm{Ti}+\mathrm{LiCl} 5$
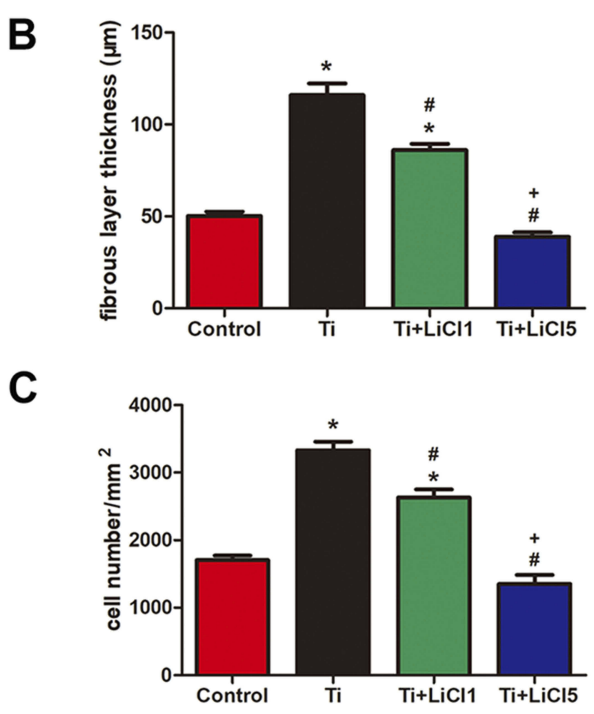

D

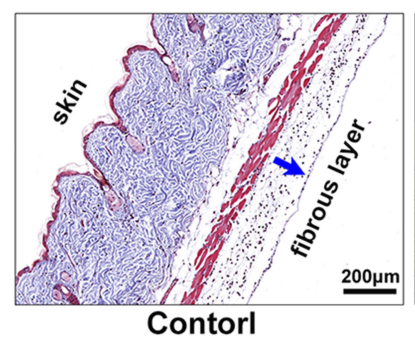

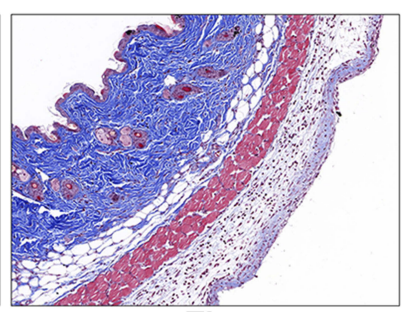

$\mathrm{Ti}$

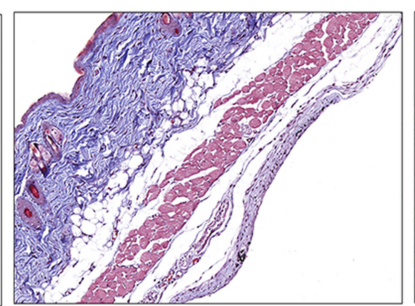

$\mathrm{Ti}+\mathrm{LiCl1}$

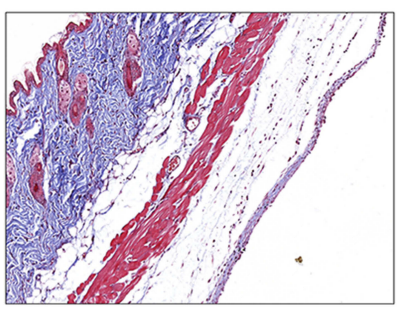

$\mathrm{Ti}+\mathrm{LiCl5}$

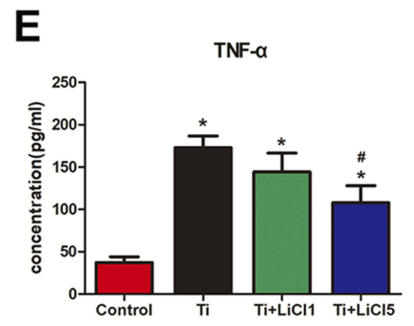

$\mathbf{F}$

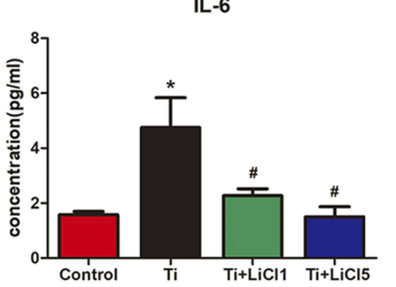

G

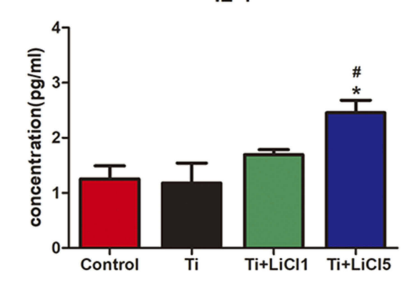

H

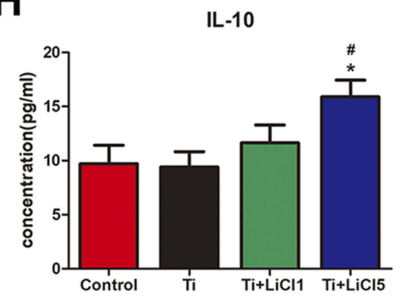

Figure 8 Images of hematoxylin eosin and Masson trichrome staining on the air pouch tissues at day 4.

Notes: (A) hematoxylin eosin stained images showing a whole structure and layers of the air-pouch tissues. (B) Thickness of fibrous layer. (C) Numbers of infiltration cells. (D) Masson's trichrome stained images obviously displaying the fibrous tissues and the infiltration cells. Cytokines in the air-pouch exudates were evaluated by ELISA: (E) TNF- $\alpha$; (F) IL-6; (G) IL-4; and (H) IL-10. scale bar: $200 \mu \mathrm{m}$. (*\# and + represent P<0.05 when compared with Control, Ti, and Ti+LiClI respectively).

Abbreviations: ELISA, enzyme-linked immunosorbent assay; TNF- $\alpha$, tumor necrosis factor alpha; IL, interleukin.

attention. Therefore, we explored the immunomodulatory ability of $\mathrm{LiCl}$ on $\mathrm{Ti}$ particle-treated macrophages and its osteogenic effects on rBMSCs.

Macrophages are versatile cells that play a crucial role in response to microenvironmental signals. Previous studies have demonstrated that macrophages can polarize into either the M1 or M2 phenotype, which represent extremes of a continuum of functional states. ${ }^{30,38}$ However, polarization is not fixed, as macrophages are sufficiently plastic and dynamic for integrating multiple signals, such as damaged tissues, microbes, and the normal tissue environment. ${ }^{11,39}$ The morphology of macrophages suggested that wear particles stimulate obvious M1 phenotypes with many synaptic structures, whereas $\mathrm{LiCl}$ enhanced the differentiation of M2 macrophages with cone-shaped ones and decreased the number of M1-like macrophages, especially in the high-LiCl concentration group (Figure 1A). According to the cell CCK8 assay results, $\mathrm{LiCl}$ and Ti particles have no obvious cytotoxicity (Figure 1B). Then, we explored whether $\mathrm{LiCl}$ regulates macrophage polarization in vitro. The results of immunostaining showed that $\mathrm{LiCl}$ upregulated Arg-1 expression and downregulated CCR7 expression (Figure 3). Flow cytometry results were consistent with immunostaining results, and changes in proinflammatory and anti- 

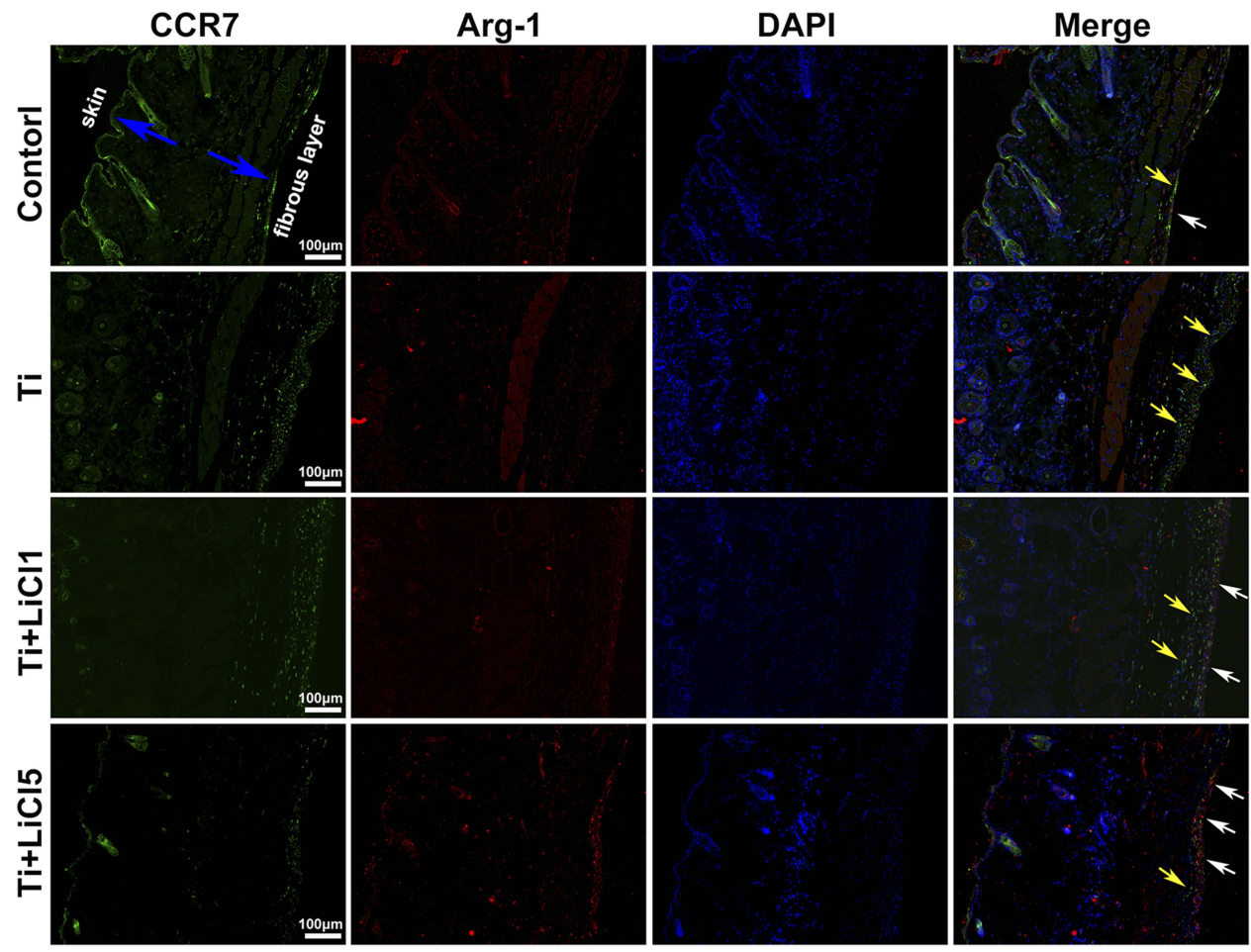

Figure 9 Images of immunofluorescence staining on air-pouch tissues at day 4 .

Notes: CCR7 (MI marker) positive cells were stained in green and Arg-I (M2 marker) positive cells were stained in red; nuclei were stained in blue; scale bar: I00 $\mu$ m. Abbreviations: CCR7, C-C chemokine receptor type 7; Arg-I, arginase-I.

inflammatory cytokines, as detected by ELISA, further reflected the functional differences between M1 and M2 phenotypes. At the genetic level, the mRNA expression of CD86 and CD163 were used by RT-PCR to further confirm the effect of $\mathrm{LiCl}$ on macrophage polarization. $\mathrm{LiCl}$ decreased CD86 expression and increased CD163 expression, which is characteristic of a downregulation of inflammatory activation and an upregulation of anti-inflammatory expression. In addition, the expression of BMP-2 and VEGF, both of which enhance tissue healing and bone formation, were also elevated in the LiCl-treated group. To further investigate the osteoimmunology of $\mathrm{LiCl}$, cell and immunofluorescence staining were performed to investigate the osteogenic differentiation of rBMSCs cultured in conditioned medium. The results obtained were consistent with the results of in vitro macrophage polarization. It has been demonstrated that osseous repair requires the coordination of diverse osteogenic and angiogenic factors during the healing cascade. $^{40}$ In summary, the results of the immune experiments indicated that $\mathrm{LiCl}$ can promote $\mathrm{M} 2$ macrophage polarization and enhance the secretion of anti-inflammatory and osteogenic cytokines. Furthermore, previous studies have already demonstrated that the immunomodulatory effects between macrophage polarization and osteogenesis may be one of the mechanisms accounting for bone regeneration. $^{41-44}$

In this study, we also investigated the wear particlestimulated inflammatory response and macrophage polarization using an air pouch model. ${ }^{9}$ The injection of Ti particles into the air pouch on the back of mice resulted in increased thickness of the fibrous layers and inflammatory cell infiltration compared to other groups. However, mice treated with $\mathrm{LiCl}$ had fewer infiltrating cells and thinner fibrous layers, suggesting a comparatively anti-inflammatory effect (Figure 8B and C). ELISA results also revealed that the $\mathrm{LiCl}$ treated group induced the production of higher levels of anti-inflammatory cytokines and lower levels of proinflammatory cytokines. Furthermore, immunofluorescence images indicated that an air pouch treated with wear particles and $5 \mathrm{mM} \mathrm{LiCl}$ presented a lower percentage of CCR7-positive cells and a higher percentage of Arg-1-positive cells than those treated with wear debris alone. The results of the in vivo air pouch model were consistent with the results obtained in vitro. 
We also provided evidence that $\mathrm{LiCl}$ inhibits the activation of ERK and p38 in Ti particle-treated macrophages. Previous studies have demonstrated that wear debris immediately stimulates the M1 phenotype bringing about a sustained inflammatory response via the MAPK pathway. ${ }^{32,45}$ Moreover, wear debris activate the MAPK pathway in bone marrow-derived macrophages, promoting the differentiation of osteoclast precursor cells. ${ }^{46}$ Furthermore, it has been reported that $\mathrm{LiCl}$ significantly suppressed LPS-induced activation of ERK phosphorylation in macrophages. ${ }^{47,48}$ Thus, MAPK pathway targeting has the capacity to regulate the two key processes resulting in aseptic loosening: wear particle-induced inflammation and osteoclast formation. Given the close relationship between $\mathrm{LiCl}$ and the MAPK pathway, we reasoned that $\mathrm{LiCl}$ might drive wear particle-treated macrophages through suppression of the ERK or p38 pathway. First, we confirmed that Ti particles activated ERK and p38 MAPKs in RAW264.7. Then, we detected that $\mathrm{LiCl}$ reduced wear particle-induced ERK and p38 phosphorylation in macrophages. These results suggested that wear particles could modulate M1 macrophage polarization and inflammatory responses in RAW264.7. Our results also revealed that $\mathrm{LiCl}$ was able to alleviate the phosphorylation of p38 and ERK, which have been shown to be concerned with wear particle-induced inflammatory responses, suggesting that $\mathrm{LiCl}$ induced macrophage polarization and inflammatory responses via the MAPK pathway (Figure 10). However, the exact molecular mechanisms by which $\mathrm{LiCl}$ influences inflammatory responses and macrophage polarization need to be subsequently investigated. In addition, this phenomenon might be one of the mechanisms underlying the immunomodulatory effects of $\mathrm{LiCl}$ on the osteogenic differentiation of rBMSCs.

\section{Conclusion}

In this study, we indicated that $\mathrm{LiCl}$ played an immunomodulatory role in macrophage polarization and $\mathrm{Ti}$ particle-stimulated inflammatory responses in vitro and in vivo, and promoted the differentiation of RAW264.7 cells to osteo-specific phenotypes by secreting osteogenic and angiogenic factors to enhance the osteogenic differentiation of rBMSCs. This immunomodulatory capacity of $\mathrm{LiCl}$ may be attributed to the inhibition of the MAPK signaling pathway. Conclusively, these results suggest that the immunomodulatory properties of $\mathrm{LiCl}$ to macrophage polarization and its subsequent effect on the osteoimmune

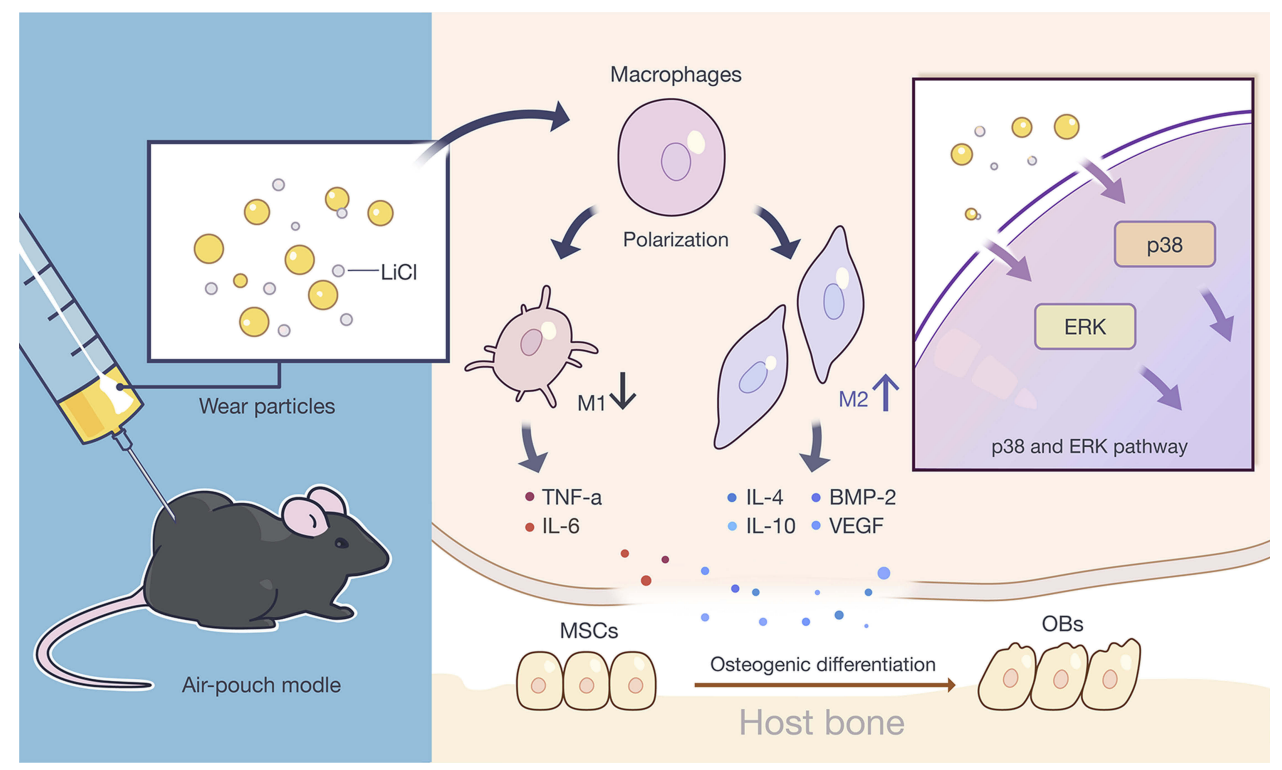

Figure $10 \mathrm{LiCl}$ modulated macrophage polarization to promote the osteogenic differentiation of $r \mathrm{BMSCs}$ via the suppression of ERK and $\mathrm{p} 38$ phosphorylation. Notes: $\mathrm{LiCl}$ exerted an immunomodulatory effect on macrophage polarization and attenuated Ti particle-induced inflammatory responses in vitro and in vivo, and generated an immune microenvironment to improve the osteogenic differentiation capability of rBMSCs. This immunomodulatory capacity of LiCl may be attributed to the inhibition of ERK and p38 signaling pathway.

Abbreviations: $\mathrm{LiCl}$, lithium chloride; rBMSCs, rat bone marrow mesenchymal stem cells; ERK, extracellular signal-regulated kinase; Ti, titanium. 
environment may be of importance to the mitigation and treatment of wear particle-induced osteolysis.

\section{Acknowledgment}

This work was supported by the National Natural Science Foundation of China (81772309), Shanghai Pujiang Talent Program (18PJD035), and Shanghai Sailing Program (18YF1418800).

\section{Author contributions}

All authors contributed to data analysis, drafting and revising the article, gave final approval of the version to be published, and agree to be accountable for all aspects of the work.

\section{Disclosure}

The authors report no conflicts of interest in this work.

\section{References}

1. Bin Abd Razak HR, Tan CS, Chen YJ, et al. Age and preoperative knee society score are significant predictors of outcomes among Asians following total knee arthroplasty. J Bone Joint Surg Am. 2016;98(9):735-741. doi:10.2106/JBJS.15.00280

2. Rao AJ, Gibon E, Ma T, et al. Revision joint replacement, wear particles, and macrophage polarization. Acta Biomater. 2012;8 (7):2815-2823. doi:10.1016/j.actbio.2012.03.042

3. Goodman SB, Gibon E, Pajarinen J, et al. Novel biological strategies for treatment of wear particle-induced periprosthetic osteolysis of orthopaedic implants for joint replacement. $J R$ Soc Interface. 2014;11(93):20130962. doi:10.1098/rsif.2013.0962

4. Gallo J, Goodman SB, Konttinen YT, Raska M. Particle disease: biologic mechanisms of periprosthetic osteolysis in total hip arthroplasty. Innate Immun. 2013;19(2):213-224. doi:10.1177/1753425912451779

5. Purdue PE, Koulouvaris P, Potter HG, Nestor BJ, Sculco TP. The cellular and molecular biology of periprosthetic osteolysis. Clin Orthop Relat Res. 2007;454:251-261. doi:10.1097/01.blo.0000238813.95035.1b

6. Nine MJ, Choudhury D, Hee AC, Mootanah R, Osman NAA. Wear debris characterization and corresponding biological response: artificial hip and knee joints. Materials (Basel). 2014;7(2):980-1016. doi:10.3390/ma7020 980

7. Yang $\mathrm{H}, \mathrm{Xu} \mathrm{Y}$, Zhu M, et al. Inhibition of titanium-particle-induced inflammatory osteolysis after local administration of dopamine and suppression of osteoclastogenesis via D2-like receptor signaling pathway. Biomaterials. 2016;80:1-10. doi:10.1016/j.biomaterials.2015.11.046

8. Ping Z, Wang Z, Shi J, et al. Inhibitory effects of melatonin on titanium particle-induced inflammatory bone resorption and osteoclastogenesis via suppression of NF-kappaB signaling. Acta Biomater. 2017;62:362-371. doi:10.1016/j.actbio.2017.08.046

9. Li B, Hu Y, Zhao Y, et al. Curcumin attenuates titanium particleinduced inflammation by regulating macrophage polarization in vitro and in vivo. Front Immunol. 2017;8:55.

10. Liu W, Li J, Cheng M, et al. Zinc-modified sulfonated polyetheretherketone surface with immunomodulatory function for guiding cell fate and bone regeneration. Adv Sci (Weinh). 2018;5(10):1800749. doi:10.1002/advs.201800749

11. Murray PJ. Macrophage Polarization. Annu Rev Physiol. 2017;79 (541-566). doi:10.1146/annurev-physiol-022516-034339
12. Kang H, Kim S, Wong DSH, et al. Remote manipulation of ligand nanooscillations regulates adhesion and polarization of macrophages in vivo. Nano Lett. 2017;17(10):6415-6427. doi:10.1021/acs.nanolett.7b03405

13. $\mathrm{Li} \mathrm{B}$, Cao H, Zhao $\mathrm{Y}$, et al. In vitro and in vivo responses of macrophages to magnesium-doped titanium. Sci Rep. 2017;7:42707. doi:10.1038/srep42707

14. Yuan X, Cao H, Wang J, et al. Immunomodulatory effects of calcium and strontium co-doped titanium oxides on osteogenesis. Front Immunol. 2017;8:1196. doi:10.3389/fimmu.2017.01196

15. Ono T, Takayanagi H. Osteoimmunology in bone fracture healing. Curr Osteoporos Rep. 2017;15(4):367-375. doi:10.1007/s11914-017-0381-0

16. Takayanagi H. Osteoimmunology: shared mechanisms and crosstalk between the immune and bone systems. Nat Rev Immunol. 2007;7 (4):292-304. doi:10.1038/nri2062

17. Takayanagi H. Osteoimmunology in 2014: two-faced immunologyfrom osteogenesis to bone resorption. Nat Rev Rheumatol. 2015;11 (2):74-76. doi:10.1038/nrrheum.2014.219

18. Hao HP, Wen LB, Li JR, et al. LiCl inhibits PRRSV infection by enhancing Wnt/beta-catenin pathway and suppressing inflammatory responses. Antiviral Res. 2015;117:99-109. doi:10.1016/j.antiviral. 2015.02.010

19. Kim S, Bong N, Kim OS, et al. Lithium chloride suppresses LPSmediated matrix metalloproteinase- 9 expression in macrophages through phosphorylation of GSK-3beta. Cell Biol Int. 2015;39 (2):177-184. doi:10.1002/cbin.10352

20. Chen HC, Chien WC, Chang MY, et al. The iNOS/Src/FAK axis contributes to lithium chloride-mediated macrophage migration. Nitric Oxide. 2015;47:58-64. doi:10.1016/j.niox.2015.04.001

21. Gu Y, Wang Z, Shi J, et al. Titanium particle-induced osteogenic inhibition and bone destruction are mediated by the GSK-3beta/betacatenin signal pathway. Cell Death Dis. 2017;8(6):e2878. doi:10.1038/cddis.2017.518

22. Qian K, Cheng X, Zhang D, et al. Antiviral effect of lithium chloride on replication of avian leukosis virus subgroup $\mathrm{J}$ in cell culture. Arch Virol. 2018;163(4):987-995. doi:10.1007/s00705-017-3692-7

23. Khan MS, Ali T, Abid MN, et al. Lithium ameliorates lipopolysaccharide-induced neurotoxicity in the cortex and hippocampus of the adult rat brain. Neurochem Int. 2017;108:343-354. doi:10.1016/j. neuint.2017.05.008

24. Leu SJ, Yang YY, Liu HC, et al. Valproic acid and lithium meditate anti-inflammatory effects by differentially modulating dendritic cell differentiation and function. J Cell Physiol. 2017;232(5):1176-1186. doi: $10.1002 /$ jcp. 25604

25. Jiang C, Xiao F, Gu X, et al. Inhibitory effects of ursolic acid on osteoclastogenesis and titanium particle-induced osteolysis are mediated primarily via suppression of NF-kappaB signaling. Biochimie. 2015;111:107-118. doi:10.1016/j.biochi.2015.02.002

26. Frellsen AF, Hansen AE, Jolck RI, et al. Mouse positron emission tomography study of the biodistribution of gold nanoparticles with different surface coatings using embedded copper-64. ACS Nano. 2016;10(11):9887-9898. doi:10.1021/acsnano.6b03144

27. Li B, Jha RK, Qi YJ, et al. Early cellular responses of BMSCs genetically modified with bFGF/BMP2 co-cultured with ligament fibroblasts in a three-dimensional model in vitro. Int $J$ Mol Med. 2016;38(5):1578-1586. doi:10.3892/ijmm.2016.2752

28. Sedgwick AD, Sin YM, Edwards JC, Willoughby DA. Increased inflammatory reactivity in newly formed lining tisssue. J Pathol. 1983;141(4):483-95. doi:10.1002/path.1711410406.

29. Cheng T, Zhang GY, Guo CJ, Zhang X. Effects of NF-kappaB inhibitor on titanium particulate-induced inflammation in a murine model. J Surg Res. 2010;162(2):225-230. doi:10.1016/j.jss.2009.03. 034

30. Wang Z, Xue K, Bai M, et al. Probiotics protect mice from CoCrMo particles-induced osteolysis. Int $J$ Nanomedicine. 2017;12:53875397. doi:10.2147/IJN.S130485 
31. Deng Z, Jin J, Wang Z, et al. The metal nanoparticle-induced inflammatory response is regulated by SIRT1 through NF-kappaB deacetylation in aseptic loosening. Int J Nanomedicine. 2017;12:3617-3636. doi:10.2147/IJN.S124661

32. Mahon OR, O'Hanlon S, Cunningham CC, et al. Orthopaedic implant materials drive M1 macrophage polarization in a spleen tyrosine kinase- and mitogen-activated protein kinase-dependent manner. Acta Biomater. 2018;65:426-435. doi:10.1016/j.actbio.2017.10.041

33. Sundfeldt M, Carlsson LV, Johansson CB, Thomsen P, Gretzer C. Aseptic loosening, not only a question of wear: a review of different theories. Acta Orthop. 2006;77(2):177-197. doi:10.1080/17453670610045902

34. Cherian JJ, Jauregui JJ, Banerjee S, Pierce T, MA M. What host factors affect aseptic loosening After THA and TKA? Clin Orthop Relat Res. 2015;473(8):2700-2709. doi:10.1007/s11999-015-4220-2

35. Hu Z, Ma C, Rong X, Zou S, Liu X. Immunomodulatory ECM-like microspheres for accelerated bone regeneration in diabetes mellitus. ACS Appl Mater Interfaces. 2018;10(3):2377-2390. doi:10.1021/ acsami.7b18458

36. Shi M, Chen Z, Farnaghi S, et al. Copper-doped mesoporous silica nanospheres, a promising immunomodulatory agent for inducing osteogenesis. Acta Biomater. 2016;30:334-344. doi:10.1016/j.actbio.2015.11.033

37. Liu W, Chen D, Jiang G, et al. A lithium-containing nanoporous coating on entangled titanium scaffold can enhance osseointegration through Wnt/beta-catenin pathway. Nanomedicine. 2018;14(1):153164. doi:10.1016/j.nano.2017.09.006

38. Hao S, Meng J, Zhang Y, et al. Macrophage phenotypic mechanomodulation of enhancing bone regeneration by superparamagnetic scaffold upon magnetization. Biomaterials. 2017;140:16-25. doi:10. 1016/j.biomaterials.2017.06.013

39. Zhang H, Lin C, Zeng C, et al. Synovial macrophage M1 polarisation exacerbates experimental osteoarthritis partially through R-spondin2. Ann Rheum Dis. 2018;77(10):1524-1534. doi:10.1136/annrheumdis-2018-213450
40. Wei F, Zhou Y, Wang J, Liu C, Xiao Y. The immunomodulatory role of BMP-2 on macrophages to accelerate osteogenesis. Tissue Eng Part A. 2018;24(7-8):584-594. doi:10.1089/ten.TEA.2017.0232

41. Saldana L, Valles G, Bensiamar F, et al. Paracrine interactions between mesenchymal stem cells and macrophages are regulated by 1,25-dihydroxyvitamin D3. Sci Rep. 2017;7(1):14618. doi:10.1038/ s41598-017-15217-8

42. Pan H, Xie Y, Zhang Z, et al. Immunomodulation effect of a hierarchical macropore/nanosurface on osteogenesis and angiogenesis. Biomed Mater. 2017;12(4):045006. doi:10.1088/1748-605X/aa6b7c

43. Zhang W, Zhao F, Huang D, et al. Strontium-substituted submicrometer bioactive glasses modulate macrophage responses for improved bone regeneration. ACS Appl Mater Interfaces. 2016;8(45):3074730758. doi:10.1021/acsami.6b10378

44. Li H, Lu Y, Qian J, et al. Human osteoclasts are inducible immunosuppressive cells in response to T cell-derived IFN-gamma and CD40 ligand in vitro. J Bone Miner Res. 2014;29(12):2666-2675. doi:10. 1002/jbmr.2294

45. Liu N, Meng J, Wang Z, et al. Autophagy mediated $\operatorname{TiAl}(6) \mathrm{V}(4)$ particle-induced peri-implant osteolysis by promoting expression of TNF-alpha. Biochem Biophys Res Commun. 2016;473(1):133-139. doi:10.1016/j.bbrc.2016.03.065

46. Rakshit DS, Ly K, Sengupta TK, et al. Wear debris inhibition of antiosteoclastogenic signaling by interleukin-6 and interferon-gamma. Mechanistic insights and implications for periprosthetic osteolysis. $J$ Bone Joint Surg Am. 2006;88(4):788-799. doi:10.2106/JBJS.E.00711

47. Raghavendra PB, Lee E, Parameswaran N. Regulation of macrophage biology by lithium: a new look at an old drug. $J$ Neuroimmune Pharmacol. 2014;9(3):277-284. doi:10.1007/s11481013-9516-y

48. Hull M, Lee E, Lee T, et al. Lithium chloride induces TNFalpha in mouse macrophages via MEK-ERK-dependent pathway. J Cell Biochem. 2014;115(1):71-80. doi:10.1002/jcb.24634
International Journal of Nanomedicine

\section{Publish your work in this journal}

The International Journal of Nanomedicine is an international, peerreviewed journal focusing on the application of nanotechnology in diagnostics, therapeutics, and drug delivery systems throughout the biomedical field. This journal is indexed on PubMed Central, MedLine, CAS, SciSearch ${ }^{\mathbb{}}$, Current Contents ${ }^{\mathbb{R}} /$ Clinical Medicine,
Journal Citation Reports/Science Edition, EMBase, Scopus and the Elsevier Bibliographic databases. The manuscript management system is completely online and includes a very quick and fair peer-review system, which is all easy to use. Visit http://www.dovepress.com/ testimonials.php to read real quotes from published authors. 